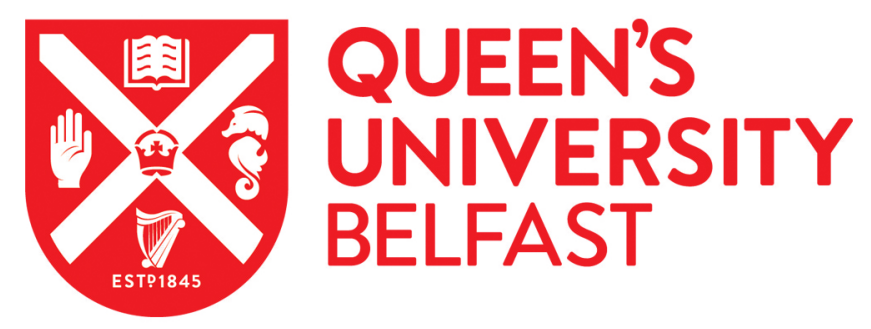

\title{
Antimicrobial efficacy of tobramycin polymeric nanoparticles for Pseudomonas aeruginosa infections in cystic fibrosis: formulation, characterisation and functionalisation with dornase alfa (DNase).
}

Deacon, J., Abdelghany, S. M., Quinn, D. J., Schmid, D., Megaw, J., Donnelly, R. F., Jones, D. S.,

Kissenpfennig, A., Elborn, J. S., Gilmore, B. F., Taggart, C. C., \& Scott, C. J. (2015). Antimicrobial efficacy of tobramycin polymeric nanoparticles for Pseudomonas aeruginosa infections in cystic fibrosis: formulation, characterisation and functionalisation with dornase alfa (DNase). Journal of Controlled Release, 198, 55-61. https://doi.org/10.1016/j.jconrel.2014.11.022

Published in:

Journal of Controlled Release

Document Version:

Peer reviewed version

Queen's University Belfast - Research Portal:

Link to publication record in Queen's University Belfast Research Portal

Publisher rights

(c) 2015 Elsevier Ltd. This manuscript version is made available under the CC-BY-NC-ND 4.0 license http://creativecommons.org/licenses/by$\mathrm{nc}-\mathrm{nd} / 4.0 /$, which permits distribution and reproduction for non-commercial purposes, provided the author and source are cited.

\section{General rights}

Copyright for the publications made accessible via the Queen's University Belfast Research Portal is retained by the author(s) and / or other copyright owners and it is a condition of accessing these publications that users recognise and abide by the legal requirements associated with these rights.

\section{Take down policy}

The Research Portal is Queen's institutional repository that provides access to Queen's research output. Every effort has been made to ensure that content in the Research Portal does not infringe any person's rights, or applicable UK laws. If you discover content in the Research Portal that you believe breaches copyright or violates any law, please contact openaccess@qub.ac.uk. 


\title{
Title
}

Antimicrobial efficacy of tobramycin polymeric nanoparticles for Pseudomonas aeruginosa infections in cystic fibrosis: formulation, characterisation and functionalisation with dornase alfa (DNase).

\section{Authors}

Jill Deacon ${ }^{\mathrm{a}}$, Sharif M Abdelghany ${ }^{\mathrm{b} 1}$, Derek J Quinn ${ }^{\mathrm{a}}$, Daniela Schmid ${ }^{\mathrm{b}}$, Julianne Megaw ${ }^{\mathrm{b}}$, Ryan F Donnelly ${ }^{\mathrm{b}}$, David S Jones ${ }^{\mathrm{b}}$, Adrien Kissenpfennig ${ }^{\mathrm{a}}$, J Stuart Elborn ${ }^{\mathrm{a}}$, Brendan F Gilmore $^{\mathrm{b}}$, Clifford C Taggart ${ }^{\mathrm{a}}$, Christopher J Scott ${ }^{\mathrm{b}^{*}}$

\section{Addresses}

${ }^{a}$ Centre for Infection and Immunity, Health Sciences Building, School of Medicine, Dentistry and Biomedical Sciences, Queen's University Belfast, 97 Lisburn Road, Belfast. BT9 7AE. Northern Ireland.

bSchool of Pharmacy, Queen's University Belfast, 97 Lisburn Road, Belfast. BT9 7BL. Northern Ireland.

${ }^{1}$ Present address: Department of Pharmaceutics and Pharmaceutical technology, Faculty of Pharmacy, University of Jordan, Queen's Rania St. Amman, 11942, Jordan.

*Corresponding Author: Christopher Scott. School of Pharmacy, Queen's University Belfast, 97 Lisburn Road, Belfast. BT9 7BL. Northern Ireland. Tel: +442890 972350 Email: c.scott@qub.ac.uk

\section{Running Title}

DNase functionalised tobramycin nanoparticles

\begin{abstract}
Inhaled antibiotics, such as tobramycin, for the treatment of Pseudomonas aeruginosa pulmonary infections are associated with the increase in life expectancy seen in cystic fibrosis (CF) patients over recent years. However, the effectiveness of this aminoglycoside is still limited by its inability to penetrate the thick DNA-rich mucus in the lungs of these patients, leading to low antibiotic exposure to resident bacteria. In this study, we created novel polymeric nanoparticle (NP) delivery vehicles for tobramycin. Using isothermal titration calorimetry, we showed that tobramycin binds with alginate polymer and, by exploiting this interaction, optimised the production of tobramycin alginate/chitosan NPs. It was established that NP antimicrobial activity against $P$. aeruginosa PA01 was equivalent to unencapsulated tobramycin (minimum inhibitory concentration $0.625 \mathrm{mg} / \mathrm{L}$ ). Galleria mellonella was employed as an in vivo model for $P$. aeruginosa infection. Survival rates of 90\% were observed following injection of NPs, inferring low NP toxicity. After infection with $P$. aeruginosa, we showed that a lethal inoculum was effectively cleared by tobramycin NPs in a dose dependent manner. Crucially, a treatment with NPs prior to infection provided a longer window of antibiotic protection, doubling survival rates from $40 \%$ with free tobramycin to $80 \%$ with NP treatment. Tobramycin NPs were then functionalised with dornase alfa (recombinant human deoxyribonuclease I, DNase), demonstrating DNA degradation and improved NP penetration of CF sputum. Following incubation with CF sputum, tobramycin NPs both with and without DNase functionalisation, exhibited antipseudomonal effects. Overall, this work demonstrates the production of effective antimicrobial NPs, which may have clinical utility as mucus-penetrating tobramycin delivery vehicles, combining two widely used $\mathrm{CF}$ therapeutics into a single NP formulation. This nano-antibiotic represents a strategy to overcome the mucus barrier, increase local drug
\end{abstract}


concentrations, avoid systemic adverse effects and improve outcomes for pulmonary infections in $\mathrm{CF}$.

\section{Keywords}

Tobramycin; alginate; nanoparticle; Pseudomonas aeruginosa; DNase; cystic fibrosis sputum

\section{Introduction}

Chronic pulmonary infection with pathogenic bacteria, such as Pseudomonas aeruginosa, is a life-limiting factor in patients suffering from cystic fibrosis (CF) [1]. The CF lung is compromised by the build up of purulent viscous mucus secretions, which impairs mucociliary clearance and supports bacterial infection. This bacterial presence stimulates the release of neutrophil chemoattractants from epithelial cells and neutrophils, leading to further neutrophilia and increased viscosity of mucus; due to the release of DNA following neutrophil breakdown [2-4]. This exopolysaccharide and DNA-rich biopolymer network of mucus has significant impact on the success of antibiotic treatment in these patients.

An important antibiotic used for CF lung infections caused by the Gram negative organism P. aeruginosa, is the aminoglycoside tobramycin [5-7]. Aerosolised delivery of tobramycin has been clinically adopted to alleviate the disadvantages of systemic administration, such as the narrow therapeutic index, low sputum concentrations and doselimiting nephrotoxicity and ototoxicity $[8,9]$. Recent development of tobramycin into a dry powder inhaler device Tobramycin Inhalation Powder ${ }^{\mathrm{TM}}$ (TIP), which has similar efficacy to the nebulised inhalation solution, has the advantages of improved convenience, portability and reduced treatment time burden [9-11]. However, the efficacy of tobramycin in CF patients is still limited by the ability to achieve sufficient levels at the site of infection. This can be attributed to rapid clearance of free drug, poor mucus and biofilm penetration and inactivation of the drug through binding interactions in these environments [12-14]. Therefore, novel strategies for improving tobramycin delivery could enhance the activity of this vital antibiotic.

Key benefits of nanoparticle (NP) based antibiotic formulations for use in infectious diseases include prolonged retention at the target site and diminished systemic exposure leading to reduced risk of serious dose-related side effects associated with this drug class $[12,15,16]$. In this report, we outline the optimisation strategy and formulation considerations for tobramycin loaded alginate/chitosan NPs and demonstrate antimicrobial efficacy using in vitro and in vivo models of $P$. aeruginosa infection. Furthermore, to overcome the mucus barrier, we investigate a strategy for the utilisation of this nano-platform to deliver the antibiotic more effectively to the site of infection in the CF lung by conjugation of dornase alfa (recombinant human deoxyribonuclease I, DNase) to the tobramycin NPs. It was hypothesised that DNase, which reduces mucus viscoelasticity by cleavage of DNA [4], may improve antibacterial activity by enhancing drug penetration of the mucus network. Therefore, we undertook preliminary studies to explore the effects of DNase tobramycin NPs in CF sputum samples to determine DNase activity, mucus penetration and overall antimicrobial susceptibility.

\section{Methods}

\section{Isothermal titration calorimetry}

A MicroCalorimeter iTC200 (GE Healthcare Life Sciences, UK) was used to investigate the interaction between sodium alginate (alginic acid sodium salt, Sigma-Aldrich, UK) and tobramycin by isothermal titration calorimetry (ITC) using a previously developed method [17]. Briefly, an initial injection $(0.1 \mu \mathrm{L})$ of $9 \mathrm{mM}$ tobramycin followed by 24 injections of $0.3 \mu \mathrm{L}$ tobramycin were titrated into the sample cell filled with $15 \mathrm{mM}$ alginate with 3 minute 
spacing between injections at a reference power $10 \mu \mathrm{Cal} / \mathrm{sec}$. The final figure and thermodynamic parameters were produced by subtracting the heats of dilution of tobramycin into water then fitted using the one set of sites model in the data analysis software MicroCal Origin $^{\circledR}$. The free energy of binding $(\Delta \mathrm{G})$ was calculated using the equation $\Delta G=\Delta H-$ $T \Delta S$, where $\Delta \mathrm{H}$ is the enthalpy change, $\mathrm{T}$ is temperature (Kelvin) and $\Delta \mathrm{S}$ is the change in entropy.

\section{Formulation and optimisation of tobramycin alginate/chitosan nanoparticles}

Solutions of sodium alginate ( $\mathrm{pH} 5)$, tobramycin and chitosan (low molecular weight, SigmaAldrich) (1\% acetic acid, $\mathrm{pH} 4.8)$ were prepared in ultrapure water. Tobramycin was added to alginate $(3 \mathrm{ml})$, stirred for 10 minutes then chitosan $(1 \mathrm{ml})$ was added dropwise via a $25 \mathrm{G}$ needle with pulsatory sonication over approximately 8 minutes. The NP suspension was stirred for 30 minutes, centrifuged at $16800 \mathrm{~g}$, washed twice for a total of 45 minutes and the final NP pellet was re-suspended in $1 \mathrm{ml}$ phosphate buffered saline (PBS). NP optimisation is outlined in Table 1. Volumes were kept constant for each formulation and weight ratios in $\mathrm{mg}$ of alginate:chitosan:tobramycin were varied. NP batches were prepared, observed aggregate formation recorded and if no aggregates formed, NP batches were characterised by Zetasizer (Zetasizer Nano ZS, Malvern Instruments Ltd, UK) measurements.

Triplicate NP batches were diluted in PBS and measurements performed in duplicate, recording size (mean particle size $\mathrm{Z}_{\mathrm{ave}}$ ), polydispersity index (PDI) and zeta potential. Scanning electron microscopy was performed by drying small droplets $(10 \mu \mathrm{L})$ of the final optimised formulation of tobramycin NPs on aluminium stubs, which were sputter-coated with gold and visualised (Jeol 6500 field emission gun, Japan). Stability studies were performed at $37^{\circ} \mathrm{C}$ with shaking at $120 \mathrm{rpm}$, measuring the size and PDI in PBS daily over 96 hours. For the tobramycin release study, $2 \mathrm{ml}$ of tobramycin NPs were injected via a $25 \mathrm{G}$ needle into a Slide-A-Lyzer ${ }^{\circledR}$ Dialysis Cassette 7000 MW (Thermo Scientific, UK) placed into $28 \mathrm{ml}$ PBS reservoir, in a shaking incubator at $37^{\circ} \mathrm{C}$. Samples of $200 \mu \mathrm{L}$ were taken from the PBS reservoir at specified time points over 48 hours and tobramycin content was analysed compared to standards prepared in PBS.

For tobramycin NPs, drug loading was calculated by analysis of tobramycin in the supernatants obtained during the wash steps. Calibration curves were prepared using known concentrations of tobramycin dissolved in the supernatant fluid obtained during washing of blank NPs. In this way, any interference was accounted for at each concentration. Based on a protocol of aminoglycoside detection [18] the product fluorescence was measured at 380/460 $\mathrm{nm}$ (FLUOstar Optima, BMG Labtech) and the reagent consisted of $80 \mathrm{mg}$ phthaldialdehyde dissolved in $1 \mathrm{ml}$ ethanol, $0.2 \mathrm{ml}$ diethyl ether, $0.2 \mathrm{ml}$ boric acid $0.4 \mathrm{M} \mathrm{pH} 9.7$ and $0.4 \mathrm{ml} 2$ mercaptoethanol (all reagents Sigma-Aldrich).

\section{Antimicrobial activity}

Broth microdilution tests were performed to determine the minimum inhibitory concentration (MIC) of tobramycin against $P$. aeruginosa PA01. An overnight PA01 suspension in LuriaBertani (LB) broth was adjusted to optical density (OD) $0.3(570 \mathrm{~nm})$ and diluted 1:1000 to give approximately $5 \times 10^{5} \mathrm{cfu} / \mathrm{ml}$. This inoculum $(100 \mu \mathrm{L})$ was added to $100 \mu \mathrm{L}$ of tobramycin NPs and free tobramycin serially diluted two fold in LB broth from a starting concentration of $20 \mathrm{mg} / \mathrm{L}$. Positive and negative growth controls and blank NPs at equivalent polymer concentrations were included. Following 18 hours incubation under shaking at $37^{\circ} \mathrm{C}$, the absorbance at $570 \mathrm{~nm}$ was measured to determine the MIC as the lowest concentration at which no visible bacterial growth occurred (OD less than 0.15).

Tobramycin NPs, free tobramycin $(250 \mu \mathrm{g} / \mathrm{ml})$ and PBS controls were tested alone for toxic effects in Galleria mellonella (livefoodsdirect.co.uk). P. aeruginosa (PA01) infections 
were treated (i) 30 minutes following a lethal infection (NP 2.5, 25 and $250 \mu \mathrm{g} / \mathrm{ml}$ and free $250 \mu \mathrm{g} / \mathrm{ml}$ tobramycin) (ii) pre-treatment (NP and free $250 \mu \mathrm{g} / \mathrm{ml}$ tobramycin) 96 hours prior to lethal infection. For each experiment, 10 larvae were injected with $20 \mu \mathrm{L}$ into the last left pro-leg using a $29 \mathrm{G}$ needle. A second injection was into the last right pro-leg. Uninfected controls were of two sterile PBS injections resulting in 90-100\% survival in each experiment (data not shown). Percentage survival was plotted over 96 hours by recording the number of dead larvae during incubation at $37^{\circ} \mathrm{C}$ in the dark. Time zero, $\mathrm{t}=0$, was the point of last injection during the toxicity screen or time of infection. Overnight PA01 cultures were grown in LB broth, centrifuged at $4000 \mathrm{~g}$ for 30 minutes, re-suspended in PBS to an OD 1.0 at 570 $\mathrm{nm}$ and diluted to give a lethal dose after 24 hours (approximately $10^{3} \mathrm{cfu} / \mathrm{ml}$ ).

\section{DNase tobramycin NPs}

EDC (1-ethyl-3-(3-dimethylaminopropyl)carbodiimide, Sigma-Aldrich) (3mg) was stirred with pre-formulated tobramycin NPs for 5 minutes then $0.5 \mathrm{ml} \mathrm{DNase}(1 \mathrm{mg} / \mathrm{ml}$ Pulmozyme ${ }^{\circledR}$, Roche, UK) was added and stirred overnight. NPs were centrifuged for 30 minutes at $16800 \mathrm{~g}$ and re-suspended in $1 \mathrm{ml}$ PBS.

Rhodamine loaded DNase tobramycin NPs were formulated by adding $250 \mu \mathrm{L}$ of 2 $\mathrm{mg} / \mathrm{ml}$ rhodamine $6 \mathrm{G}$ (Sigma-Aldrich) to the alginate solution. Rhodamine loaded no drug NPs (prepared without tobramycin and DNase) were used as controls. Rhodamine content was determined against a calibration curve prepared from rhodamine added to blank NPs (without rhodamine) immediately after formulation (time 0 ) and following 24 hours at room temperature to determine leaching of the dye from NPs. At each time point NPs were centrifuged for 30 minutes and washed in PBS.

A standard protocol of bicinchoninic acid protein assay (BCA Protein Assay Kit, Thermo Scientific Pierce, UK) was followed to quantify DNase present in NPs against standards of known amounts of DNase added to blank NPs or rhodamine loaded no drug NPs as appropriate.

\section{NP evaluation in cystic fibrosis sputum}

Sputum from CF patients was obtained anonymously from the Adult CF centre at Belfast City Hospital. Sputum samples were in excess to requirements for diagnostic purposes. Permission to use sputum samples, which would have been disposed of, for validation purposes was given by the Director of R\&D in Belfast Health and Social Care Trust. Sputum was frozen immediately at $-80^{\circ} \mathrm{C}$ and used the next day after thawing at room temperature.

For DNA degradation evaluation, $50 \mu \mathrm{g} / \mathrm{ml}$ DNase, free and conjugated to tobramycin NPs, blank NPs and PBS were incubated with CF sputum (ratio sputum:treatment 5:1) for 2 hours at $37^{\circ} \mathrm{C}$, then visualised by ethidium bromide staining and agarose gel electrophoresis.

The penetration of rhodamine loaded DNase tobramycin NPs and rhodamine loaded no drug NPs through CF sputum was determined, based on a previously developed model [19]. A layer $(0.25 \mathrm{ml})$ of $10 \%$ gelatin (Porcine type A, Sigma-Aldrich) was set in a 48 well microplate. To this, $0.25 \mathrm{ml}$ sputum or PBS control was added and allowed to settle, then 0.1 $\mathrm{ml}$ rhodamine loaded NPs were added. NPs were diluted to give DNase concentration of 50 $\mu \mathrm{g} / \mathrm{ml}$ and rhodamine loaded no drug NPs were diluted to provide equivalent rhodamine concentrations. After 24 hours at room temperature, the sputum/NP layer was removed, gelatin washed, melted and fluorescence measured at $480 / 520 \mathrm{~nm}$. To calculate the percentage NPs penetrated through sputum, the fluorescence values of the gelatin in corresponding controls without a sputum layer were defined as $100 \%$ NP penetration. Duplicate measurements were carried out in three separate experiments.

Treatment groups of DNase tobramycin NPs, tobramycin NPs, a mixture of tobramycin NPs plus free DNase, free tobramycin and PBS were tested against CF sputum 
samples. Sputum samples were mixed thoroughly and a ratio of sputum:treatment 5:1 was incubated with shaking at $37^{\circ} \mathrm{C}$ for 24 hours. Samples were vortexed before serial tenfold dilutions in PBS were plated onto Pseudomonas isolation agar (Difco ${ }^{\mathrm{TM}}, \mathrm{BD}, \mathrm{UK}$ ) for viable counting. DNase tobramycin NPs were undiluted and other treatments made at equivalent drug concentrations. Results were normalised to the mean cfu/ml of the untreated PBS control, represented as percentage survival of $P$. aeruginosa. PBS controls ranged from $6 \mathrm{x}$ $10^{6}$ to $6 \times 10^{8} \mathrm{cfu} / \mathrm{ml}$.

\section{Statistical analysis}

Results were analysed with GraphPad Prism, version 5.03, GraphPad Software (San Diego, USA). G. mellonella survival shown as Kaplan-Meier plots and analysis was performed using the log rank (Mantel-Cox) test for significance. As detailed in figure legends, t-tests and one way ANOVA analyses were conducted. Statistical significance critical values were defined as $* \mathrm{P}<0.05, * * \mathrm{P}<0.01$.

\section{Results}

Formulation and optimisation of tobramycin alginate/chitosan nanoparticles

Given that tobramycin has a net positive charge, it was hypothesised that its properties were conducive to formulation into alginate-based NPs. Prior to NP formulation, this potential binding was studied through ITC. Tobramycin was injected into an alginate solution and, as shown in Figure 1, an exothermic reaction was observed, indicating binding between the two molecules. The values in the inset table calculated from Origin data analysis showed a dissociation affinity constant $\left(\mathrm{K}_{\mathrm{D}}\right)$ in the micromolar range $(1.62 \mu \mathrm{M})$. The negative enthalpy change $(\Delta \mathrm{H}=-40.33 \mathrm{kcal} / \mathrm{mol})$ indicated a favourable reaction and the calculated entropy component $(-\mathrm{T} \Delta \mathrm{S})$ was positive and classified as unfavourable. However, the larger enthalpy compensated for this entropy and the free energy $(\Delta \mathrm{G})$ was also favourable, calculated to be $7.98 \mathrm{kcal} / \mathrm{mol}$. The negative free energy showed the process was enthalpically driven and indicated strong binding.

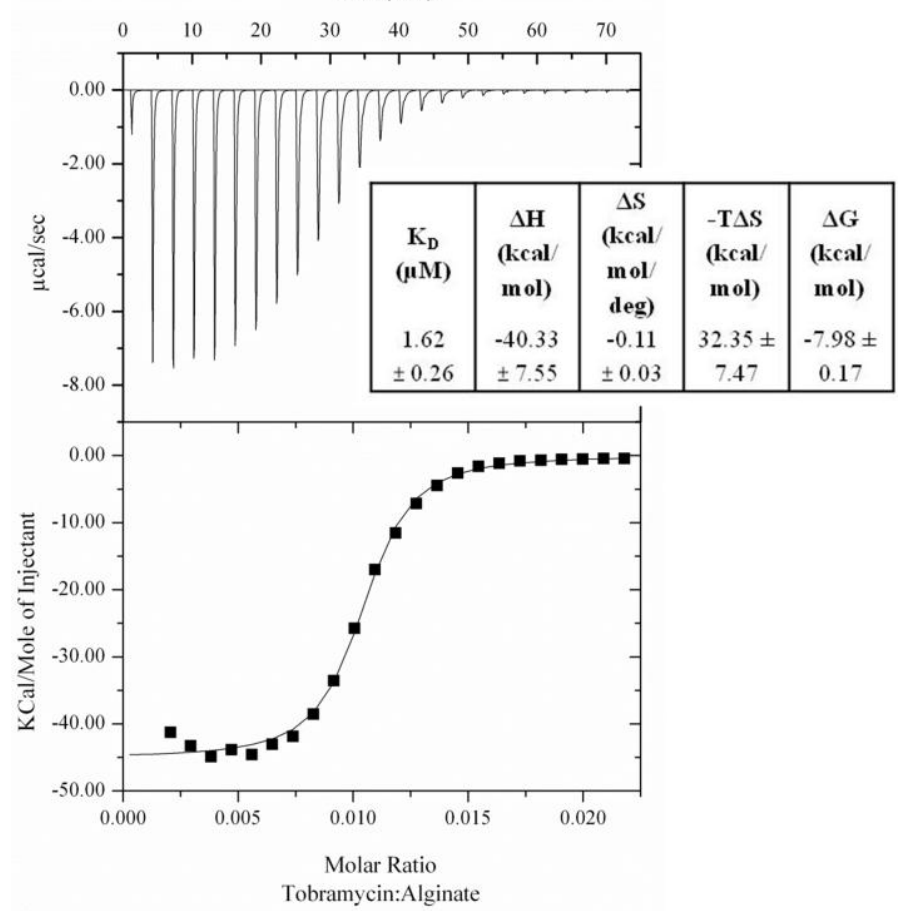

Figure 1 
Having confirmed the ability of tobramycin to interact with alginate, NP formulations using chitosan as a stabilising polymer were investigated whereby alginate and tobramycin were first mixed, followed by the addition of chitosan. This process has been referred to as nanoplex formation via self-assembly of polyelectrolytes [20]. Consistent with this previous work we observed the counterion (chitosan) is essential and a critical balance of the components was required for NP formation. Various parameters were investigated to arrive at an optimal formulation by altering the proportions of the three components (alginate, tobramycin and chitosan) as highlighted in Table 1.

Table 1. Optimisation of the formulation of tobramycin alginate/chitosan nanoparticles.

\begin{tabular}{|c|c|c|c|}
\hline Formulation & $\begin{array}{l}\text { Component weight ratio }(\mathrm{mg}) \\
\text { alginate : chitosan : tobramycin }\end{array}$ & $\begin{array}{c}\text { Aggregates? } \\
\mathrm{Y}, \mathrm{N}^{\mathrm{a}}\end{array}$ & $\begin{array}{c}\text { NP size range } \\
<1000 \mathrm{~nm} ? \\
\mathrm{~N}^{\mathrm{b}} \mathrm{A}^{\mathrm{b}}, \mathrm{Y}, \mathrm{N}\end{array}$ \\
\hline 1 & $4: 0.75: 1.5$ & $\mathrm{Y}$ & N/A \\
\hline 2 & $4: 1.5: 1.5$ & $\mathrm{Y}$ & N/A \\
\hline 3 & $6: 1.5: 1.5$ & $\mathbf{N}$ & $\mathbf{Y}$ \\
\hline 4 & $6: 3: 1.5$ & $\mathrm{Y}$ & N/A \\
\hline 5 & $6: 1.5: 3$ & $\mathrm{~N}$ & $\mathrm{~N}$ \\
\hline 6 & $6: 1.5: 6$ & $\mathrm{Y}$ & N/A \\
\hline 7 & $9: 1.5: 1.5$ & $\mathbf{N}$ & $\mathbf{Y}$ \\
\hline 8 & $9: 1.5: 3$ & $\mathrm{Y}$ & N/A \\
\hline
\end{tabular}

${ }^{\mathrm{a}} \mathrm{Y}, \mathrm{N}:$ Yes or No.

${ }^{b}$ N/A: Not Applicable, size not measured due to aggregate formation.

Two formulations chosen for further characterisation were 3 and 7 (Table 2). A low PDI of 0.19 indicated the NP populations had a uniform size distribution and the zeta potentials were negative. Tobramycin content for formulation 3 was $91.7 \mu \mathrm{g} / \mathrm{mg}$ polymer whereas formulation 7 resulted in a lower drug loading of $41.1 \mu \mathrm{g} / \mathrm{mg}$ polymer. Therefore formulation 3 was chosen for further work using the ratio of alginate:chitosan:tobramycin 6:1.5:1.5.

Table 2. Tobramycin NP characterisation ${ }^{\mathrm{a}}$.

\begin{tabular}{|c|c|c|c|c|c|c|}
\hline $\begin{array}{l}\text { Tobramycin } \\
\text { NP } \\
\text { formulation }\end{array}$ & $\begin{array}{l}\text { Component weight ratio } \\
\text { (mg) alginate : chitosan } \\
\text { tobramycin }\end{array}$ & Size (nm) & PDI & $\begin{array}{l}\text { Zeta } \\
\text { potential } \\
(\mathrm{mV})\end{array}$ & $\begin{array}{l}\text { Tobramycir } \\
\text { content } \\
\left(\mu \mathrm{g} / \mathrm{mg}^{\mathrm{b}}\right. \\
\text { polymer })\end{array}$ & $\begin{array}{l}\text { Encapsulation } \\
\text { efficiency }^{c} \\
(\%)\end{array}$ \\
\hline 3 & $6: 1.5: 1.5$ & $\begin{array}{r}505 \\
\pm 32 \\
\end{array}$ & $\begin{array}{c}0.19 \\
\pm 0.06 \\
\end{array}$ & $\begin{array}{l}-25.7 \\
\pm 0.6\end{array}$ & $\begin{array}{c}91.7 \\
\pm 18.1\end{array}$ & $\begin{array}{l}45.8 \\
\pm 7.0\end{array}$ \\
\hline 7 & $9: 1.5: 1.5$ & $\begin{array}{r}538 \\
\pm 43 \\
\end{array}$ & $\begin{array}{c}0.19 \\
\pm 0.03 \\
\end{array}$ & $\begin{array}{l}-28.0 \\
\pm 1.2\end{array}$ & $\begin{array}{c}41.1 \\
\pm 11.4\end{array}$ & $\begin{array}{r}20.6 \\
\pm 5.7 \\
\end{array}$ \\
\hline
\end{tabular}

${ }^{a} \mathrm{n}=3$ batches prepared on separate days, mean \pm standard deviation.

${ }^{\mathrm{b}}$ Polymer weight includes total of alginate and chitosan weights.

c(tobramycin amount in NP/tobramycin amount added initially)*100. 
The mean diameter measured by Zetasizer analysis of $505 \mathrm{~nm}$ was confirmed by scanning electron microscopy, highlighting spherical morphology of NPs (Figure 2 a). Under agitated conditions at $37^{\circ} \mathrm{C}$, size and PDI of NPs were retained over 96 hours (Figure $2 \mathrm{~b}$ ) and the tobramycin release study highlighted a typical biphasic release of the drug with $45 \%$ released in the first 90 minutes, which increased to $80 \%$ after 48 hours (Figure $2 \mathrm{c}$ ). This indicated that although the drug is able to interact with the alginate during formulation to achieve good encapsulation, it can still be successfully released.

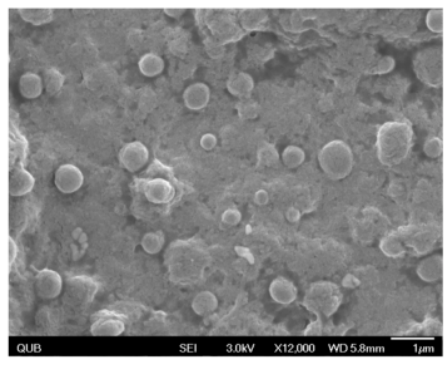

Figure 2 (b)

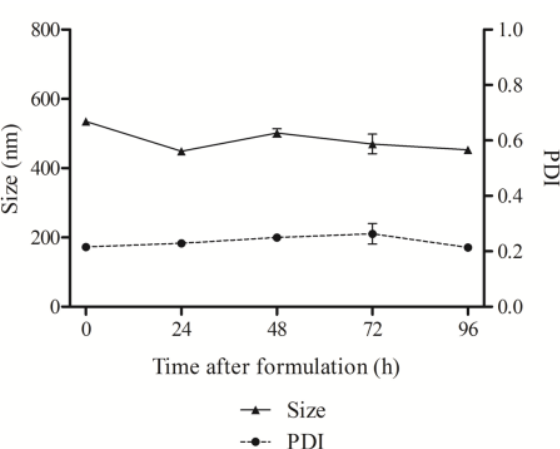

(c)

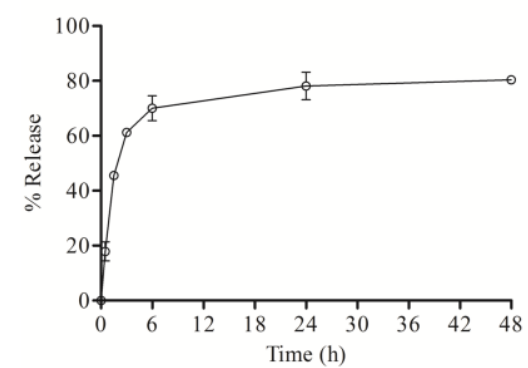

Antimicrobial activity of tobramycin nanoparticles

To examine the therapeutic effects of the nano-formulated tobramycin, the MIC was established against $P$. aeruginosa (PA01) (Figure 3). Tobramycin NPs demonstrated comparable antimicrobial effects to unencapsulated tobramycin: both MICs were $0.625 \mathrm{mg} / \mathrm{L}$. This indicated that the potency of tobramycin had not been adversely affected by formulation processes. Again, despite the previously confirmed interaction with alginate, tobramycin was able to exert its antimicrobial effect. At equivalent NP polymer concentrations examined, blank NPs had no discernible effects on the visible growth of the bacteria.

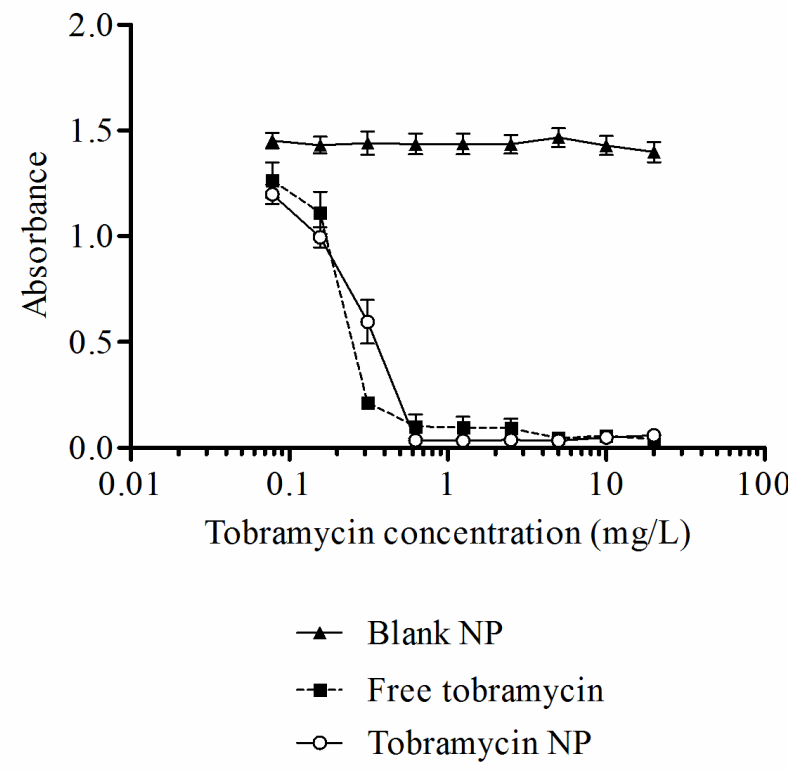

Figure 3

The antimicrobial effects of the NPs were then evaluated in more detail towards PA01 infection in vivo using a Galleria mellonella model. As a model for infection, it has strong 
evidence as an appropriate alternative method for studying novel antimicrobials with clinical relevance $[21,22]$. Firstly, the potential toxicity of tobramycin NPs was examined. No significant difference in survival was found between the NP-treated and untreated arms $(90 \%$ survival, 96 hours, Figure 4 a). Next, the ability of the drug formulations to treat infectioninduced mortality was measured with bacteria $\left(1.4 \times 10^{3} \mathrm{cfu} / \mathrm{ml}\right)$ administered at $\mathrm{t}=0$ and drug treatment 30 minutes later. G. mellonella was sensitive to this low inoculum of PA01 as untreated larvae did not survive more than 24 hours, similar to previous responses with other P. aeruginosa strains [22,23]. Tobramycin prevented mortality in the larvae in a dose dependent manner. The NP dose of $2.5 \mu \mathrm{g} / \mathrm{ml}$ tobramycin did not enhance survival of infected G. mellonella, resulting in the same response as for the untreated group. With increasing doses of tobramycin, survival increased, where $25 \mu \mathrm{g} / \mathrm{ml}$ and $250 \mu \mathrm{g} / \mathrm{ml}$ showed $40 \%$ and $90 \%$ survival respectively (Figure $4 \mathrm{~b}$ ). These survival plots also showed that administration of free and NP formulated tobramycin $(250 \mu \mathrm{g} / \mathrm{ml})$ generated similar therapeutic benefit. It was hypothesised that the controlled release nature of the NPs could provide an extended therapeutic window. Therefore, a prophylactic study was prepared where the larvae were treated with either free or NP formulated tobramycin. PA01 bacteria were then introduced after an incubation period of 96 hours ( $t=0$ post infection) and mortality was measured over a further 96 hours. The resulting effect was a significant enhancement of survival upon tobramycin NP treatment compared to the free drug, as shown in Figure $4 \mathrm{c}$. NPs offered a protective effect, providing $80 \%$ survival whereas the free drug showed only half this value at $40 \%$ survival after 96 hours.

(a)

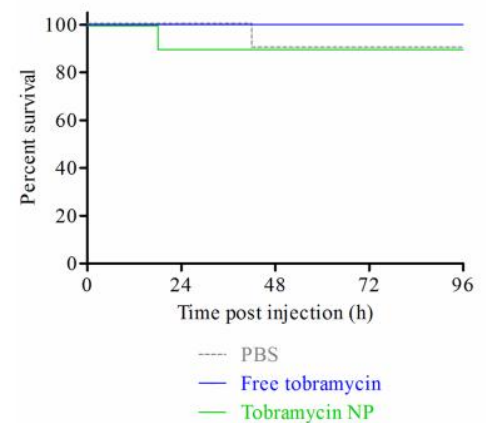

Figure 4 (b)

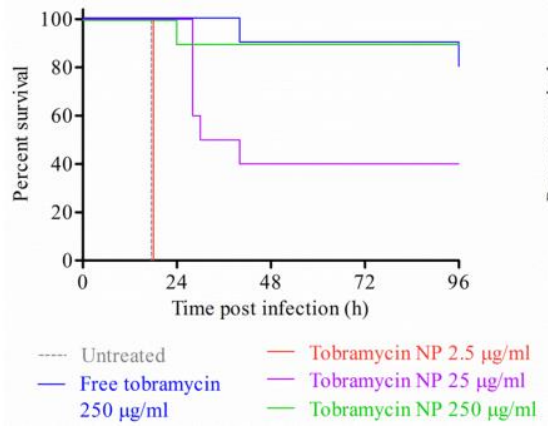

(c)

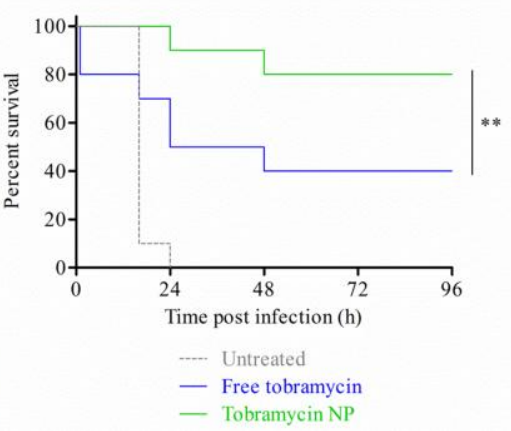

DNase functionalisation of tobramycin nanoparticles

DNase functionalisation was facilitated by the covalent crosslinking of DNase to tobramycin NPs using carbodiimide chemistry. These NPs were characterised as before (Table 3) producing negatively charged particles of a mean diameter $457 \mathrm{~nm}$ and polydispersity index 0.21 . Tobramycin loading was $69.6 \mu \mathrm{g} / \mathrm{mg}$ polymer, DNase content was $15.7 \mu \mathrm{g} / \mathrm{mg}$ polymer. Against PA01 cultures, DNase tobramycin NPs showed similar antimicrobial effects to the tobramycin NPs with a single point increase in MIC to $1.25 \mathrm{mg} / \mathrm{L}$ (supplementary figure 1). 
Table 3. DNase tobramycin NP characterisation ${ }^{\mathrm{a}}$.

\begin{tabular}{ccccccc}
\hline $\begin{array}{c}\text { Size } \\
(\mathrm{nm})\end{array}$ & PDI & $\begin{array}{c}\text { Zeta } \\
\text { potential } \\
(\mathrm{mV})\end{array}$ & $\begin{array}{c}\text { Tobramycin } \\
\text { content } \\
\left(\mu \mathrm{g} / \mathrm{mg}^{\mathrm{b}}\right. \\
\left.\text { polymer }^{\mathrm{b}}\right)\end{array}$ & $\begin{array}{c}\text { Tobramycin } \\
\text { encapsulation } \\
\text { efficiency } \\
(\%)^{\mathrm{c}}\end{array}$ & $\begin{array}{c}\text { DNase } \\
\text { content } \\
(\mu \mathrm{g} / \mathrm{mg} \\
\left.\text { polymer }^{\mathrm{b}}\right)\end{array}$ & $\begin{array}{c}\text { MIC } \\
\text { against } \\
(\mathrm{mg} / \mathrm{L})\end{array}$ \\
\hline $\begin{array}{c}\text { P57 } \\
\pm 12\end{array}$ & 0.21 & -23.0 & 69.6 & 34.8 & 15.7 & 1.25 \\
\hline 0.01 & \pm 1.5 & \pm 6.4 & \pm 3.5 & \pm 2.7 & 1.25 \\
\hline
\end{tabular}

${ }^{a} \mathrm{n}=3$ batches prepared on separate days, mean \pm standard deviation.

${ }^{b}$ Polymer weight includes total of alginate and chitosan weights.

c(tobramycin amount in NP/tobramycin amount added initially)*100.

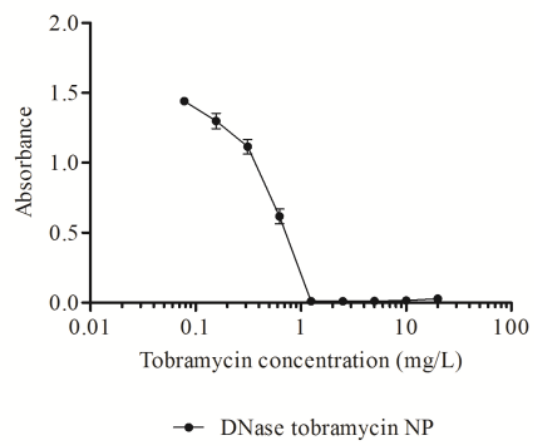

\section{Supplementary figure 1}

It was hypothesised that functionalisation of tobramycin NPs with DNase, which reduces mucus viscoelasticity by DNA cleavage [4], may improve the antibacterial activity of tobramycin by enhancing drug penetration of the mucus network. As there was no suitable preclinical in vivo model available to reflect the viscous and infectious mucus environment in the CF lung, the efficacy of the NPs towards infected clinical CF sputum was examined instead. DNase activity was confirmed through its ability to degrade DNA found in CF sputum samples (Figure 5 a). DNA in CF sputum that was sham treated with PBS and blank NPs showed a characteristic smear of DNA. This was diminished after DNase treatment, indicating degradation of the high molecular weight DNA present. Crucially, the DNase functionalised NPs also degraded DNA, highlighting the ability of the formulation to break down DNA in clinical isolates. To ensure that NPs could penetrate the CF sputum and were not totally obstructed by the mesh network, a sputum penetration assay was employed. Using rhodamine 6G loaded NPs it was found that, following 24 hours incubation, the percentage of DNase tobramycin NPs transported across the sputum layer was significantly enhanced over the permeation of their dye loaded no drug NP equivalent (Figure $5 \mathrm{~b}$ ). However it was also observed that these no drug NPs were not entirely obstructed and still had $30 \%$ penetration. Rhodamine loading of NPs was quantified at 0 and 24 hours and there was no detectable difference in the amount of rhodamine in NPs (supplementary figure 2), indicating any change in fluorescence was due to the presence of NPs in the gelatin layer, not leaching of the dye.

Finally, in a small group of sputum samples, we examined the antimicrobial efficacy of the tobramycin delivery vehicles. Untreated sputum samples had varying levels of $P$. aeruginosa ranging from $6 \times 10^{6}$ to $6 \times 10^{8} \mathrm{cfu} / \mathrm{ml}$. All samples showed sensitivity to tobramycin as depicted in Figure $5 \mathrm{c}$, which also exemplifies the difference in responses between samples. For example, all tobramycin treatments of sample 4 resulted in very large bacterial kill, with less than 5\% P. aeruginosa survival, whereas when sample 3 was treated with DNase tobramycin NPs there was $50 \%$ survival of bacteria. This was to be expected due 
to the heterogeneous patient population. Despite this, we found that in comparison to the other treatments, the DNase conjugated tobramycin NPs were equally effective and in two of four samples trended towards improved efficacy.

(a)

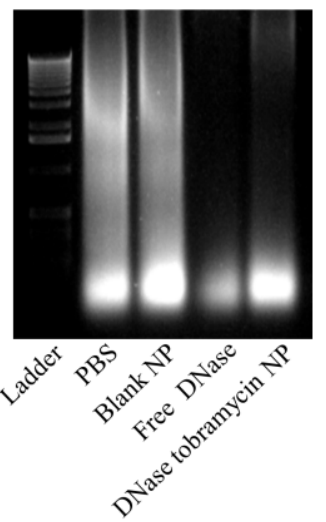

Figure 5 (b)

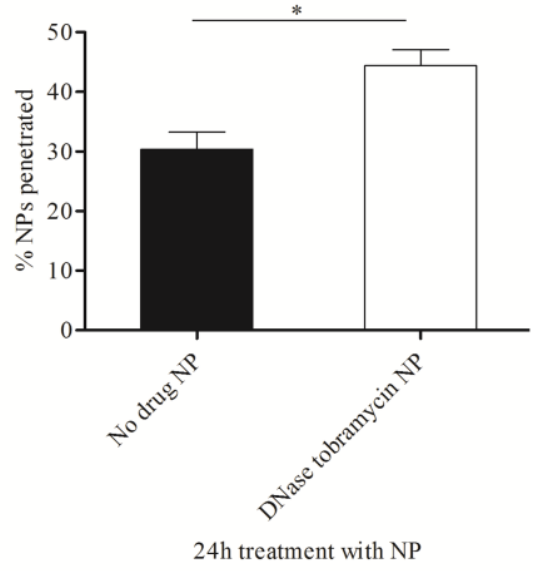

(c)

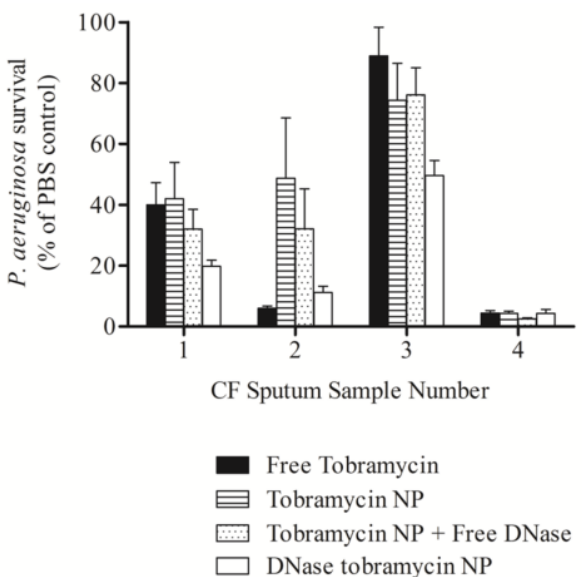

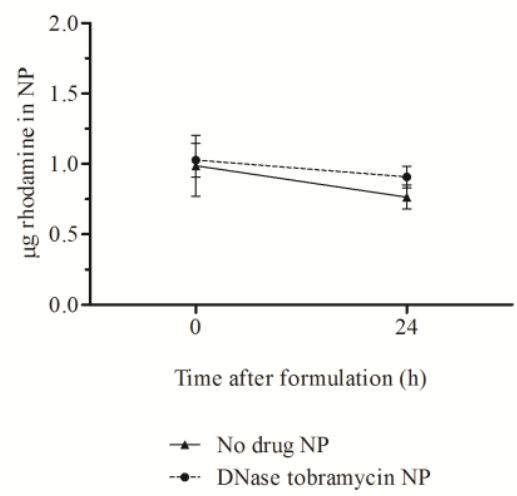

Supplementary figure 2

\section{Discussion}

Polymeric NP delivery of antibiotics has the potential to provide prolonged residence time at sites of infection, yield sustained therapeutic release and present site-specific targeting $[12,15,24,25]$. In this current work we developed an alginate-based NP system for tobramycin. This polymer has been shown to favour the encapsulation of hydrophilic drugs [17] and it was thought that the cationic nature of tobramycin would improve entrapment efficiency. Thermodynamic analysis of ITC data confirmed a strong interaction between alginate and tobramycin. The thermodynamic profile observed was similar to previous work with aminoglycosides [26] and alginate [27], where the favourable enthalpy suggested that hydrogen bonding and van der Waals interactions were the main forces dictating the interactions.

Exploiting this tobramycin-alginate interaction, we successfully fabricated alginate/chitosan NPs with high drug loading, which is frequently a limitation of preparation of antibiotic NP formulations [20]. Alginate has been shown to improve tobramycin encapsulation [19] and, in comparison to these poly(lactide-co-glycolide) acid (PLGA) NPs, the formulation developed here requires minimal organic solvent use. In addition to no requirements of extreme conditions during formulation, our NP production lends itself to economic and environmental efficiency. The $\mathrm{pH}(4.8-5)$ and mixing order of the NP components determines the mechanism of formation of the NPs. The first step was to mix the 
large polysaccharide alginate with the oppositely charged molecule tobramycin and, as verified by ITC, interactions occurred allowing for ionic crosslinking. Upon addition of the large polyelectrolyte chitosan, the crosslinking was reinforced by further electrostatic interactions forming a polyelectrolyte complex [28]. The formation of NPs via the polyelectrolyte complexation of negatively charged alginate with positively charged chitosan has been exploited for a variety of drugs whose optimal formulation ratio of components proved similar to our studies [17,29-31]. In agreement with other studies $[20,32,33]$ drug loading did not simply correlate with increasing polymer content during NP formulation, highlighting the need for empirical determination and optimisation.

The efficacy of the high-drug loaded tobramycin NPs was evaluated using the $G$. mellonella infection model. This model has been shown to have positive correlation with mammalian models in determining virulence against $P$. aeruginosa [23] and its use as an infection model has many advantages over conventional mammalian models, such as low cost, ease of experimentation and ethical acceptability [34]. Antibiotic susceptibilities, pharmacokinetic and pharmacodynamic properties have been successfully determined in $G$. mellonella and it proves to be valuable in assessing antimicrobials $[21,22,35]$. The use of an invertebrate model with a functioning immune system provides further insight and allows refinement of the efficacy of novel antimicrobial delivery systems at this early stage in the drug development process. We have shown here that tobramycin NPs were able to rescue larvae from a lethal infection of PA01. The comparable antimicrobial efficacy of NPs and free tobramycin reflected the similar MIC values. This is in agreement with the previously shown correlation between in vitro and in vivo sensitivities [22]. Crucially, NP pre-treatment results in this systemic model proved superior to free tobramycin. This suggests that the NP delivery system acts as an antibiotic depot providing sustained antibiotic concentrations above the MIC, which is desirable for improving therapeutic outcomes in infections. The normal pharmacokinetic clearance mechanisms of free drugs in vivo may have been overcome by NPs, highlighting a major advantage of NP therapy, as we have observed previously [25].

An important parameter for targeting infections residing in the lungs of $\mathrm{CF}$ patients is effective penetration of the barrier posed by lung mucus. Viscoelastic CF mucus contains raised levels of DNA and actin from neutrophils, pathogens and epithelial cells [2]. These components, combined with the ability of bacteria to grow in biofilms, create a potent barrier to treatment of colonised lungs [14,36-38]. In this report, we have focused on DNA as a barrier in CF sputum. Clinically, DNase (Pulmozyme) is inhaled to improve lung function and clearance and to reduce the viscosity of the mucus in CF lungs $[3,4,39]$. By functionalising DNase to NPs, we showed cleavage of DNA in CF sputum and demonstrated this enhanced NP penetration, providing evidence for a barrier effect of DNA. Previously the use of adjuvant DNase treatments with NPs has been assessed [37,40,41] but the work here represents the first attempt to functionalise NPs with active DNase.

Antimicrobial efficacy of DNase tobramycin NPs was shown against $P$. aeruginosa, with slight variation in the MIC compared to tobramycin NPs, similar to previous work [19]. In our initial evaluation of antimicrobial activity in CF sputum samples, we found heterogeneity in the bacterial load and this may have impaired the ability to produce significantly enhanced therapeutic effects with the DNase functionalised NPs. Nonetheless, the results show that NPs are at least as efficacious as unencapsulated tobramycin and in future studies with larger cohorts of matched patient samples these effects may be more apparent.

\section{Conclusion}


The development of mucus penetrating drug carriers which offer sustained levels of therapeutics at mucosal surfaces has great potential. In this investigation we have shown the optimisation strategy of an alginate/chitosan nanoparticle drug delivery system for tobramycin. We demonstrated the efficacy of tobramycin NPs against $P$. aeruginosa infections both in vitro and in vivo in the Galleria mellonella model. We further showed the versatility of tobramycin NPs by designing dual modality NPs incorporating DNase, which showed effective penetration and anti-pseudomonal activity in CF patient sputum. The results presented here further support the utility of nano-antibiotic technology and in agreement with recent literature [12], our tobramycin polymeric NPs have high antibiotic loading, stability and mucus penetration abilities, which are the unmet desirable properties for such a delivery system for CF pulmonary infection therapies. Successful delivery of antimicrobials to difficult-to-penetrate sites of chronic infection is essential to improve therapeutic outcomes in $\mathrm{CF}$ lung infections and it is hoped future drug design would consider barriers to drug delivery, efficacy and patient adherence. Our polymeric nano-antibiotics represent a promising strategy in the battle to treat pulmonary infection in the hostile environment of the CF lungs.

\section{Acknowledgements}

This work was funded in part by the Engineering and Physical Sciences Research Council (EP/H031065/1).

\section{References}

[1] J. Lyczak, C. Cannon, G. Pier, Lung infections associated with cystic fibrosis, Clin. Microbiol. Rev. 15 (2002) 194-222. doi:10.1128/CMR.15.2.194.

[2] J. V. Fahy, B.F. Dickey, Airway mucus function and dysfunction., N. Engl. J. Med. 363 (2010) 2233-47. doi:10.1056/NEJMra0910061.

[3] M.E. Hodson, Aerosolized dornase alfa (rhDNase) for therapy of cystic fibrosis., Am. J. Respir. Crit. Care Med. 151 (1995) S70-4. doi:10.1164/ajrccm/151.3_Pt_2.S70.

[4] S. Shak, D.J. Capon, R. Hellmiss, S.A. Marsters, C.L. Baker, Recombinant human DNase I reduces the viscosity of cystic fibrosis sputum., Proc. Natl. Acad. Sci. U. S. A. 87 (1990) 9188-92. doi:10.1073/pnas.87.23.9188.

[5] A.R. Smyth, S.C. Bell, S. Bojcin, M. Bryon, A. Duff, P. Flume, et al., European cystic fibrosis society standards of care: Best practice guidelines., J. Cyst. Fibros. 13 (2014) S23-42. doi:10.1016/j.jcf.2014.03.010.

[6] P.J. Mogayzel, E.T. Naureckas, K.A. Robinson, G. Mueller, D. Hadjiliadis, J.B. Hoag, et al., Cystic Fibrosis Pulmonary Guidelines Chronic Medications for Maintenance of Lung Health., Am. J. Respir. Crit. Care Med. 187 (2013) 680-689. doi:10.1164/rccm.201207-1160OE.

[7] G. Döring, P. Flume, H. Heijerman, J.S. Elborn, Treatment of lung infection in patients with cystic fibrosis: current and future strategies., J. Cyst. Fibros. 11 (2012) 461-79. doi:10.1016/j.jcf.2012.10.004.

[8] A. Chuchalin, E. Amelina, F. Bianco, Tobramycin for inhalation in cystic fibrosis: Beyond respiratory improvements., Pulm. Pharmacol. Ther. 22 (2009) 526-32. doi:10.1016/j.pupt.2009.06.001.

[9] M.W. Konstan, D.E. Geller, P. Minić, F. Brockhaus, J. Zhang, G. Angyalosi, Tobramycin inhalation powder for P. aeruginosa infection in cystic fibrosis: The EVOLVE trial., Pediatr. Pulmonol. 46 (2011) 230-238. doi:10.1002/ppul.21356.

[10] M.W. Konstan, P.A. Flume, M. Kappler, R. Chiron, M. Higgins, F. Brockhaus, et al., Safety, efficacy and convenience of tobramycin inhalation powder in cystic fibrosis 
patients: The EAGER trial., J. Cyst. Fibros. 10 (2011) 54-61.

doi:10.1016/j.jcf.2010.10.003.

[11] M.D. Parkins, J.S. Elborn, Tobramycin Inhalation Powder ${ }^{\mathrm{TM}}$ : a novel drug delivery system for treating chronic Pseudomonas aeruginosa infection in cystic fibrosis., Expert Rev. Respir. Med. 5 (2011) 609-22. doi:10.1586/ers.11.56.

[12] K. Hadinoto, W.S. Cheow, Nano-antibiotics in chronic lung infection therapy against Pseudomonas aeruginosa., Colloids Surf. B. Biointerfaces. 116 (2014) 772-85. doi:10.1016/j.colsurfb.2014.02.032.

[13] B.E. Hunt, A. Weber, A. Berger, B. Ramsey, A.L. Smith, Macromolecular mechanisms of sputum inhibition of tobramycin activity., Antimicrob. Agents Chemother. 39 (1995) 34-39. doi:10.1128/AAC.39.1.34.

[14] B.S. Tseng, W. Zhang, J.J. Harrison, T.P. Quach, J.L. Song, J. Penterman, et al., The extracellular matrix protects Pseudomonas aeruginosa biofilms by limiting the penetration of tobramycin, Environ. Microbiol. 15 (2013) 2865-2878. doi:10.1111/1462-2920.12155.

[15] M.-H. Xiong, Y. Bao, X.-Z. Yang, Y.-H. Zhu, J. Wang, Delivery of antibiotics with polymeric particles., Adv. Drug Deliv. Rev. (2014). doi:10.1016/j.addr.2014.02.002.

[16] K. Forier, K. Raemdonck, S.C. De Smedt, J. Demeester, T. Coenye, K. Braeckmans, Lipid and polymer nanoparticles for drug delivery to bacterial biofilms., J. Control. Release. 190 (2014) 607-623. doi:10.1016/j.jconrel.2014.03.055.

[17] S.M. Abdelghany, D. Schmid, J. Deacon, J. Jaworski, F. Fay, K.M. McLaughlin, et al., Enhanced antitumor activity of the photosensitizer meso-tetra(N-methyl-4-pyridyl) porphine tetra tosylate through encapsulation in antibody-targeted chitosan/alginate nanoparticles., Biomacromolecules. 14 (2013) 302-310. doi:10.1021/bm301858a.

[18] J. Benson, P. Hare, O-phthalaldehyde: fluorogenic detection of primary amines in the picomole range. Comparison with fluorescamine and ninhydrin., Proc. Natl. Acad. Sci. U. S. A. 72 (1975) 619-622.

[19] F. Ungaro, I. d'Angelo, C. Coletta, R. d'Emmanuele Di Villa Bianca, R. Sorrentino, B. Perfetto, et al., Dry powders based on PLGA nanoparticles for pulmonary delivery of antibiotics: Modulation of encapsulation efficiency, release rate and lung deposition pattern by hydrophilic polymers., J. Control. Release. 157 (2012) 149-159. doi:10.1016/j.jconrel.2011.08.010.

[20] W.S. Cheow, K. Hadinoto, Green preparation of antibiotic nanoparticle complex as potential anti-biofilm therapeutics via self-assembly amphiphile-polyelectrolyte complexation with dextran sulfate., Colloids Surfaces B Biointerfaces. 92 (2012) 5563. doi:10.1016/j.colsurfb.2011.11.024.

[21] R.J. Thomas, K.A. Hamblin, S.J. Armstrong, C.M. Müller, M. Bokori-Brown, S. Goldman, et al., Galleria mellonella as a model system to test the pharmacokinetics and efficacy of antibiotics against Burkholderia pseudomallei., Int. J. Antimicrob. Agents. 41 (2013) 330-336. doi:10.1016/j.ijantimicag.2012.12.009.

[22] L. Hill, N. Veli, P.J. Coote, Evaluation of Galleria mellonella larvae for measuring the efficacy and pharmacokinetics of antibiotic therapies against Pseudomonas aeruginosa infection., Int. J. Antimicrob. Agents. 43 (2014) 254-261. doi:10.1016/j.ijantimicag.2013.11.001.

[23] G. Jander, L.G. Rahme, F.M. Ausubel, Positive correlation between virulence of Pseudomonas aeruginosa mutants in mice and insects., J. Bacteriol. 182 (2000) 38435.

[24] I. d'Angelo, C. Conte, M.I. La Rotonda, A. Miro, F. Quaglia, F. Ungaro, Improving the efficacy of inhaled drugs in cystic fibrosis: Challenges and emerging drug delivery strategies., Adv. Drug Deliv. Rev. 75 (2014) 92-111. doi:10.1016/j.addr.2014.05.008. 
[25] S.M. Abdelghany, D.J. Quinn, R.J. Ingram, B.F. Gilmore, R.F. Donnelly, C.C. Taggart, et al., Gentamicin-loaded nanoparticles show improved antimicrobial effects towards Pseudomonas aeruginosa infection., Int. J. Nanomedicine. 7 (2012) 4053-63. doi:10.2147/IJN.S34341.

[26] A.L. Norris, C. Ozen, E.H. Serpersu, Thermodynamics and kinetics of association of antibiotics with the aminoglycoside acetyltransferase (3)-IIIb, a resistance-causing enzyme., Biochemistry. 49 (2010) 4027-35. doi:10.1021/bi100155j.

[27] M. Ferstl, A. Strasser, H.J. Wittmann, M. Drechsler, M. Rischer, J. Engel, et al., Nanofibers resulting from cooperative electrostatic and hydrophobic interactions between peptides and polyelectrolytes of opposite charge., Langmuir. 27 (2011) 14450-14459. doi:10.1021/la202252m.

[28] S. Mizrahy, D. Peer, Polysaccharides as building blocks for nanotherapeutics., Chem. Soc. Rev. 41 (2012) 2623. doi:10.1039/c1cs15239d.

[29] P. Li, Y.-N. Dai, J.-P. Zhang, A.-Q. Wang, Q. Wei, Chitosan-alginate nanoparticles as a novel drug delivery system for nifedipine., Int. J. Biomed. Sci. 4 (2008) 221-8.

[30] T. Li, X. Shi, Y. Du, Y. Tang, Quaternized chitosan/alginate nanoparticles for protein delivery., J. Biomed. Mater. Res. Part A. 83 (2007) 383-90. doi:10.1002/jbm.a.

[31] R.C. Nagarwal, R. Kumar, J.K. Pandit, Chitosan coated sodium alginate-chitosan nanoparticles loaded with 5-FU for ocular delivery: In vitro characterization and in vivo study in rabbit eye., Eur. J. Pharm. Sci. 47 (2012) 678-685. doi:10.1016/j.ejps.2012.08.008.

[32] E. Lu, S. Franzblau, H. Onyuksel, C. Popescu, Preparation of aminoglycoside-loaded chitosan nanoparticles using dextran sulphate as a counterion., J. Microencapsul. 26 (2009) 346-354. doi:10.1080/02652040802365182.

[33] S.K. Motwani, S. Chopra, S. Talegaonkar, K. Kohli, F.J. Ahmad, R.K. Khar, Chitosansodium alginate nanoparticles as submicroscopic reservoirs for ocular delivery: Formulation, optimisation and in vitro characterisation, Eur. J. Pharm. Biopharm. 68 (2008) 513-525. doi:10.1016/j.ejpb.2007.09.009.

[34] K. Kavanagh, E.P. Reeves, Exploiting the potential of insects for in vivo pathogenicity testing of microbial pathogens., FEMS Microbiol. Rev. 28 (2004) 101-12. doi:10.1016/j.femsre.2003.09.002.

[35] G. Brackman, P. Cos, L. Maes, H.J. Nelis, T. Coenye, Quorum sensing inhibitors increase the susceptibility of bacterial biofilms to antibiotics in vitro and in vivo., Antimicrob. Agents Chemother. 55 (2011) 2655-61. doi:10.1128/AAC.00045-11.

[36] S.K. Lai, Y.Y. Wang, J. Hanes, Mucus-penetrating nanoparticles for drug and gene delivery to mucosal tissues., Adv. Drug Deliv. Rev. 61 (2009) 158-171. doi:10.1016/j.addr.2008.11.002.

[37] N.N. Sanders, S.C. De Smedt, E. Van Rompaey, P. Simoens, F. De Baets, J. Demeester, Cystic fibrosis sputum: a barrier to the transport of nanospheres., Am. J. Respir. Crit. Care Med. 162 (2000) 1905-1911. doi:10.1164/ajrccm.162.5.9909009.

[38] V.J. Broughton-Head, J.R. Smith, J. Shur, J.K. Shute, Actin limits enhancement of nanoparticle diffusion through cystic fibrosis sputum by mucolytics, Pulm. Pharmacol. Ther. 20 (2007) 708-717. doi:10.1016/j.pupt.2006.08.008.

[39] P.L. Shah, S.F. Scott, R.A. Knight, C. Marriott, C. Ranasinha, M.E. Hodson, In vivo effects of recombinant human DNase I on sputum in patients with cystic fibrosis., Thorax. 51 (1996) 119-125. doi:10.1136/thx.51.2.119.

[40] N.N. Sanders, E. Van Rompaey, S.C. De Smedt, J. Demeester, On the transport of lipoplexes through cystic fibrosis sputum., Pharm. Res. 19 (2002) 451-456. doi:10.1023/A:1015139527747. 
[41] M. Dawson, D. Wirtz, J. Hanes, Enhanced viscoelasticity of human cystic fibrotic sputum correlates with increasing microheterogeneity in particle transport., J. Biol. Chem. 278 (2003) 50393-50401. doi:10.1074/jbc.M309026200.

\section{Figure Legends}

Figure 1. Isothermal titration calorimetry: titration of tobramycin $(9 \mathrm{mM})$ into alginate $(15$ $\mathrm{mM})$. The inset table shows calculated thermodynamic parameters by Origin analysis and the equation $\Delta \mathrm{G}=\Delta \mathrm{H}-\mathrm{T} \Delta \mathrm{S}$. Figure is representative of three experiments, inset table data $\mathrm{n}=3$, mean \pm standard deviation.

Figure 2. Tobramycin NP Characteristics. (a) Scanning electron microscope image of tobramycin NPs (b) NP size and PDI measurements over 96 hours from time of formulation $\left(0\right.$ h). 3 NP batches, mean \pm SEM (c) Tobramycin NP release profile in PBS at $37^{\circ} \mathrm{C}$ over 48 hours. $n=2$, mean \pm SEM.

Figure 3. MIC determination against $P$. aeruginosa PA01: free tobramycin, tobramycin NPs and blank NPs. Blank NP points representative of the equivalent polymer concentration of the tobramycin NPs at the specific tobramycin concentration tested. MICs of both free and NP tobramycin were $0.625 \mathrm{mg} / \mathrm{L} . \mathrm{n}=3$, mean \pm SEM.

Figure 4. Galleria mellonella PA01 infection model. (a) PBS, free tobramycin and tobramycin NPs, followed by an injection of PBS (no infection) to determine NP toxicity. (b) Tobramycin treatment following lethal PA01 infection. (c) Pre-treatment protocol: PA01 infection introduced after $96 \mathrm{~h}$ incubation. $\mathrm{n}=10 \mathrm{G}$. mellonella per group, percentage survival monitored over $96 \mathrm{~h}$. Log rank (Mantel-Cox) test for significance $* * \mathrm{P}<0.01$.

Figure 5. Bioactivity of DNase tobramycin NPs in CF Sputum. (a) Gel electrophoresis image of CF sputum incubated with PBS, blank NP, free DNase and DNase tobramycin NP. Image representative of 3 sputum samples. (b) Ability of rhodamine loaded no drug NPs and rhodamine loaded DNase tobramycin NPs to penetrate CF sputum. Data presented as \% NPs penetrated compared to a no sputum (PBS layer) control, $n=3$, mean \pm SEM. Unpaired t-test $* \mathrm{P}<0.05$ (c) Treatment of CF sputum with PBS, free tobramycin, tobramycin NP, tobramycin NP and free DNase, DNase tobramycin NP. Undiluted sputum samples (3-6 replicates per sample) treated for $24 \mathrm{~h}$. Data normalised to PBS control (100\% survival), represented as percentage survival of $P$. aeruginosa $\left(\left(\mathrm{cfu} \mathrm{ml}^{-1}\right.\right.$ treatment $/$ mean $\left.\mathrm{cfu} \mathrm{ml}^{-1} \mathrm{PBS}\right)$ *100) for 4 independent samples.

Supplementary figure 1. MIC determination against $P$. aeruginosa PA01: DNase tobramycin NPs. MIC was $1.25 \mathrm{mg} / \mathrm{L}$. $\mathrm{n}=3$, mean \pm SEM.

Supplementary figure 2. Release of rhodamine from no drug and DNase tobramycin NPs loaded with rhodamine $6 \mathrm{G}$, at room temperature over $24 \mathrm{~h}$. $\mathrm{n}=2$, mean $\pm \mathrm{SEM}$. 